Supporting Information

\title{
Internal and External Flow over Laser-Textured Superhydrophobic Polytetrafluoroethylene (PTFE)
}

K. M. Tanvir Ahmmed, Christian Patience and Anne-Marie Kietzig*

McGill University, 3610 University Street, Montréal, Québec, H3A 0C5, Canada

* Tel: +1(514) 398-3302. E-mail: anne.kietzig@mcgill.ca 


\section{Flow channel measurement verification}

We calculated the theoretical pressure drop with the following equation:

$$
Q=-\frac{4}{3 \mu} \frac{\Delta P}{L} b c^{3}\left[1-\frac{6 c}{b} \sum_{k=1}^{\infty} \frac{\tanh \left(\frac{\alpha_{k} b}{c}\right)}{\alpha_{k}^{5}}\right]
$$

where $\alpha_{k}=(2 k-1) \frac{\pi}{2}, k=1,2, \ldots ; Q$ is the volumetric flowrate, $\frac{\Delta P}{L}$ is the pressure gradient, $2 b$ is the width of the channel, $2 c$ is its height, and $\mu$ is the viscosity of the liquid. This theoretical prediction is plotted along with the experimental results in Figure S1.

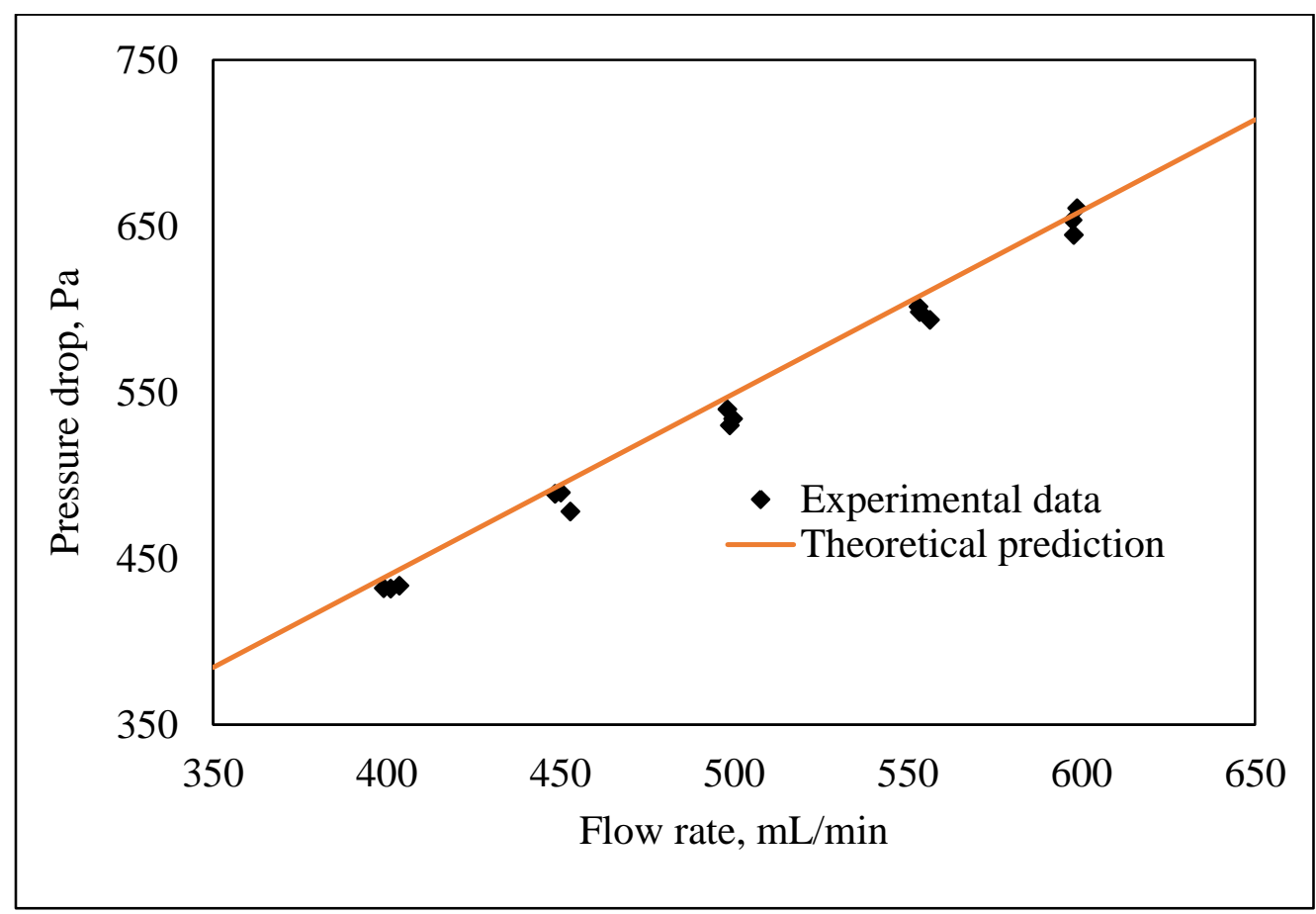

Figure S1. The accuracy of the measurements was verified in the flow channel with a non-SH flat PTFE control surface. The experimental data are from three sets of experiments. The solid curve is the theoretical prediction calculated from the physical geometry of the channel and the fluid properties. 
PTFE spheres' physical properties before and after texturing

Table S1. Physical properties of the PTFE spheres, before and after ablation.

\begin{tabular}{lll}
\hline Parameters & Original & After ablation \\
\hline Weight, g & $0.9731 \pm 0.0004$ & $0.9555 \pm 0.0012$ \\
Diameter, mm & $9.490 \pm 0.009$ & $9.450 \pm 0.012$ \\
\hline
\end{tabular}

Surface topographies of the textured PTFE surfaces.

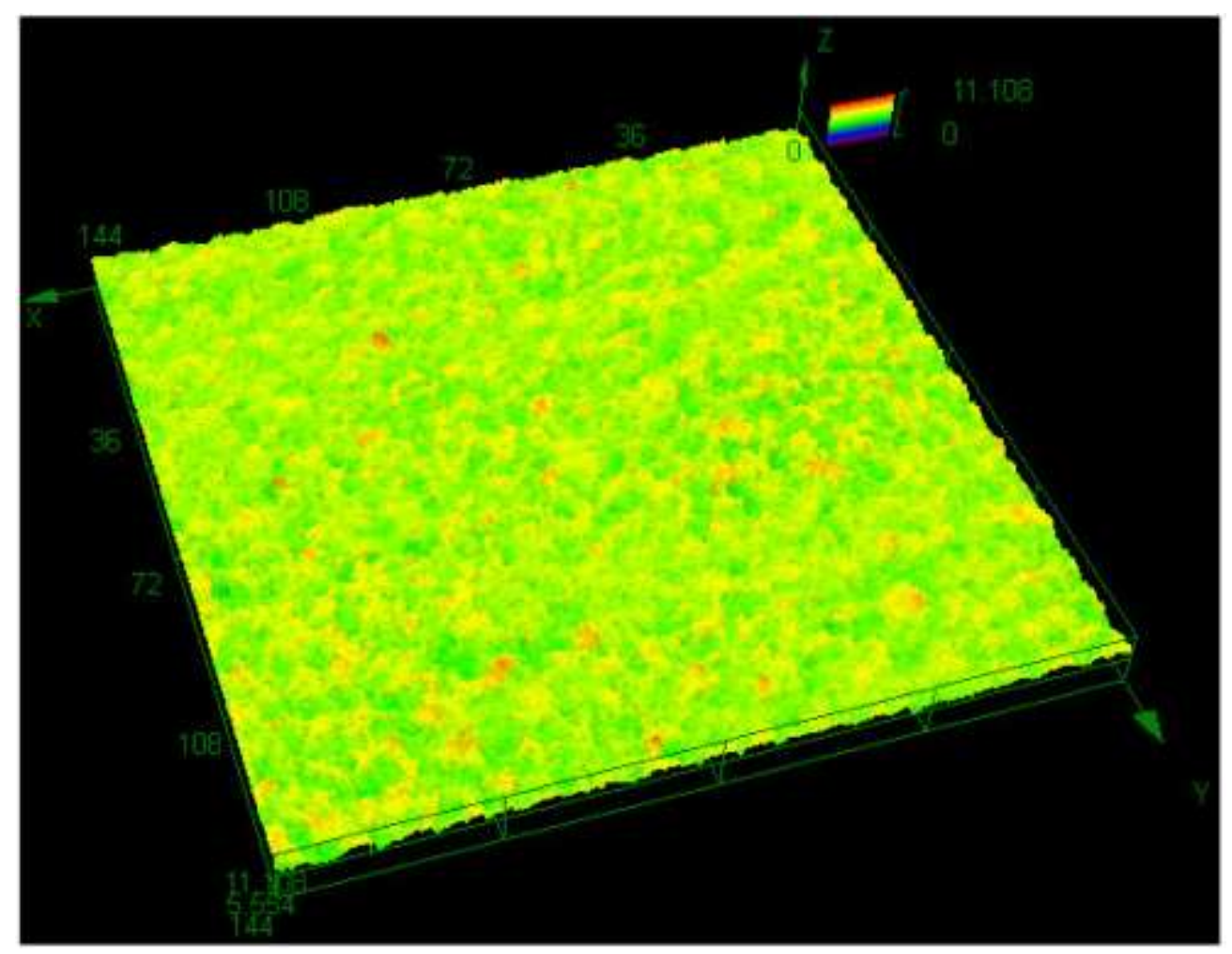

Figure S2. 3D roughness profile of the textured PTFE flat surface. Root mean square height of the surface $\mathrm{S}_{\mathrm{q}}=0.7 \mu \mathrm{m}$, maximum height of peaks, $\mathrm{S}_{\mathrm{p}}=3 \mu \mathrm{m}$, maximum height of valleys, $\mathrm{S}_{\mathrm{v}}=$ $7 \mu \mathrm{m}$. 


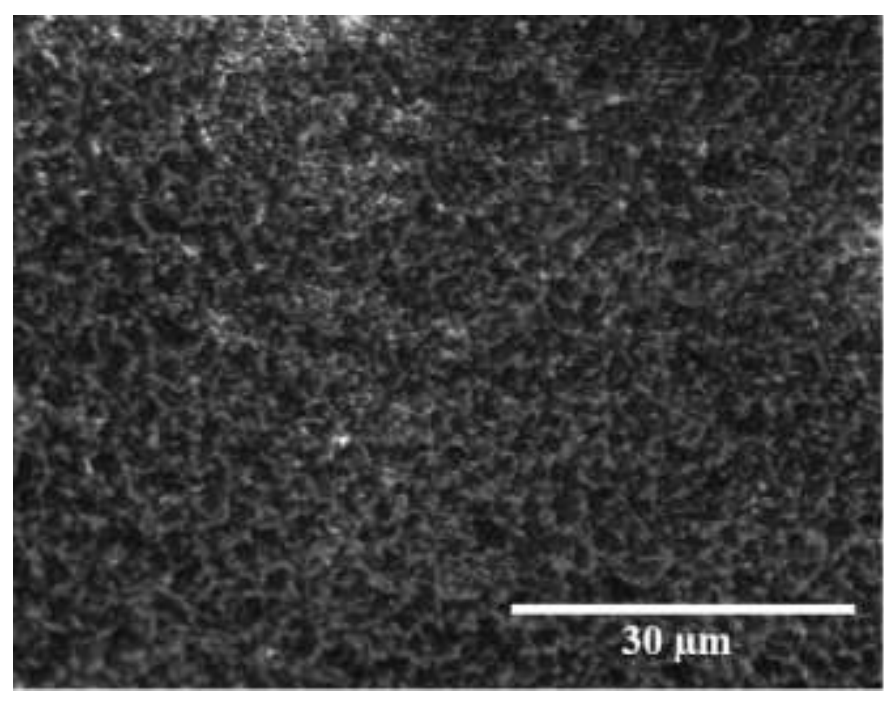

Figure S3. SEM image of the textured PTFE sphere. 
Air-water interface.

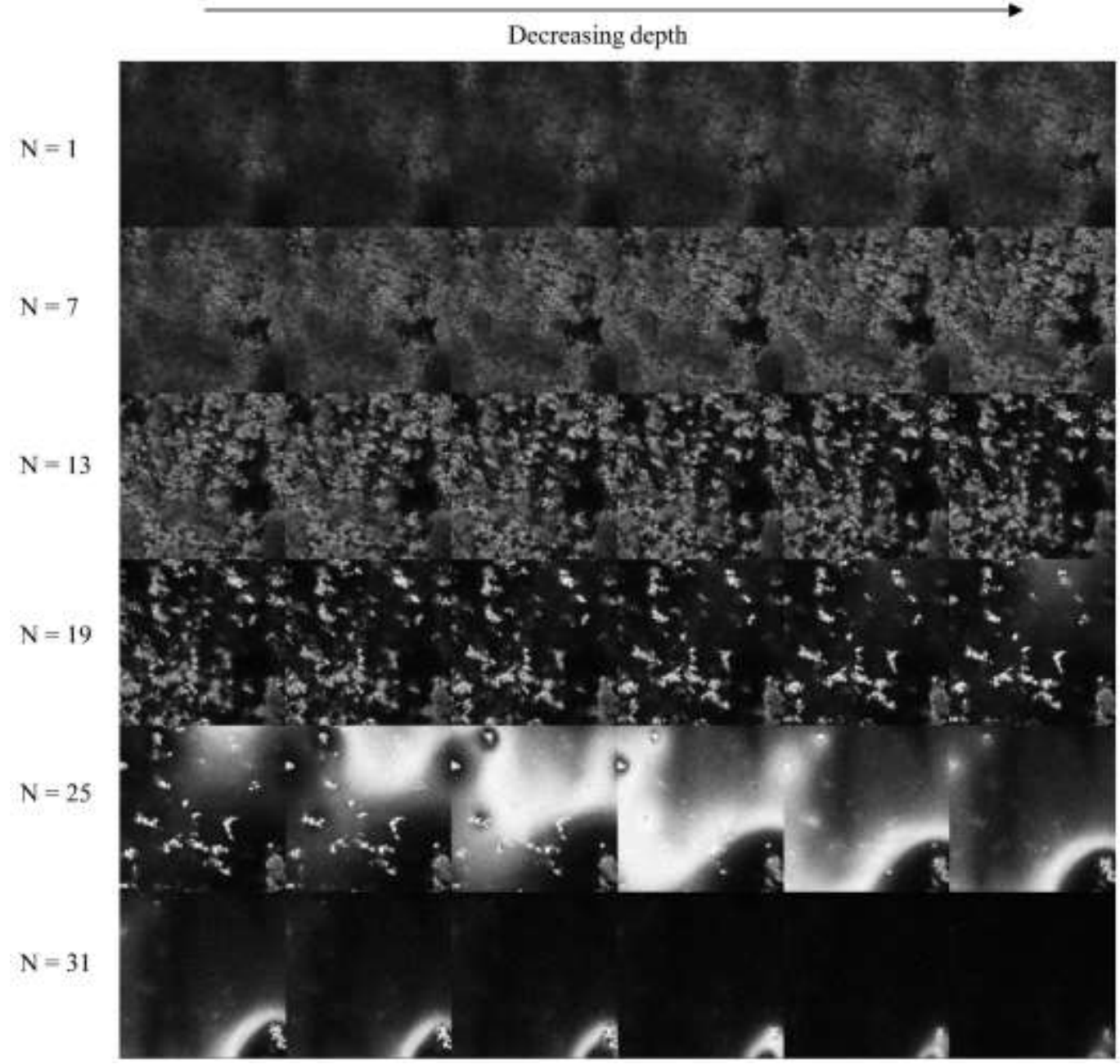

Figure S4. Montage of air-water interface determination with confocal microscopy. A total of 36 images are shown in the order of decreasing depth. $\mathrm{N}$ indicates the image number, where $\mathrm{N}=1$ is the highest depth, and $\mathrm{N}=36$ is the lowest depth. The change in depth is $1.366 \mu \mathrm{m}$ between two consecutive images. Each image is $192 \mu \mathrm{m} \times 192 \mu \mathrm{m}$ in size. 
SH and non-SH spheres falling through pure glycerol.

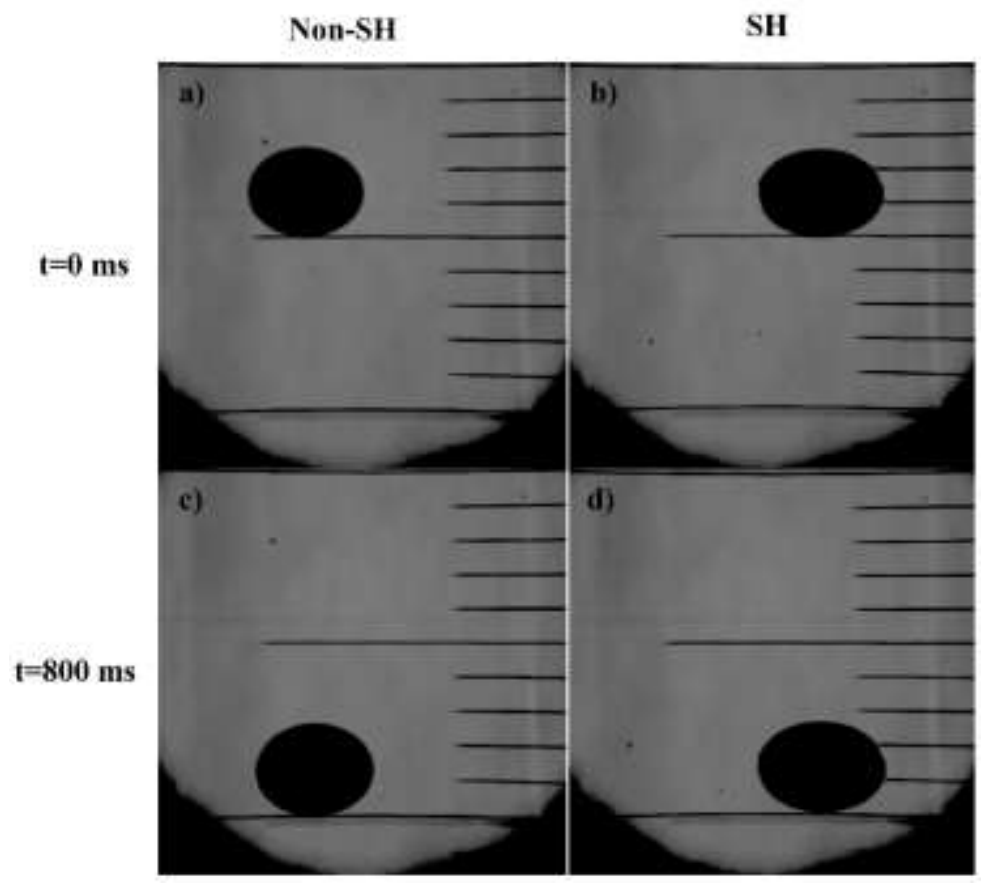

Figure S5. Two spheres falling through pure glycerol. (a) and (b) show the non-SH PTFE sphere and SH PTFE sphere, respectively. (c) and (d) show the same PTFE spheres after $800 \mathrm{~ms}$. The diameter of the spheres are $\sim 9.5 \mathrm{~mm}$. The spheres look oval because of the curvature of the cylinder. 